Draft Version OCtober 30, 2018

Preprint typeset using $\mathrm{LAT}_{\mathrm{E}} \mathrm{X}$ style emulateapj v. 03/07/07

\title{
SEARCH FOR GAMMA-RAY EMISSION FROM MAGNETARS WITH THE FERMI LARGE AREA TELESCOPE
}

A. A. Abdo ${ }^{1,2}$, M. Ackermani ${ }^{3}$, M. Ajello ${ }^{3}$, A. Allafort ${ }^{3}$, L. Baldini $^{4}$, J. Ballet $^{5}$, G. Barbiellini $^{6,7}$, M. G. Baring ${ }^{8}$, D. Bastieri ${ }^{9,10}$, R. Bellazzini ${ }^{4}$, R. D. Blandford ${ }^{3}$, E. D. Bloom ${ }^{3}$, E. Bonamente ${ }^{11,12}$, A. W. Borgland ${ }^{3}$, A. Bouvier ${ }^{3}$, J. Bregeon ${ }^{4}$, M. Brigida ${ }^{13,14}$, P. Bruel ${ }^{15}$, T. H. Burnett ${ }^{16}$, G. A. Caliandro ${ }^{17}$,

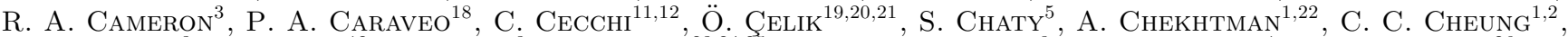
J. Chiang ${ }^{3}$, S. Ciprini ${ }^{12}$, R. Claus ${ }^{3}$, J. Conrad ${ }^{23,24,25}$, P. R. Den Hartog ${ }^{3}$, C. D. Dermer ${ }^{1}$, A. DE Angelis ${ }^{26}$, F. De Palma ${ }^{13,14}$, R. Dib ${ }^{27}$, M. Dormody ${ }^{28}$, E. do Couto E Silva ${ }^{3}$, P. S. Drell ${ }^{3}$, R. Dubois ${ }^{3}$, D. Dumora ${ }^{29}$ ' T. Enoto ${ }^{3}$, C. FAvuzzi ${ }^{13,14}$, M. Frailis ${ }^{26,30}$, P. Fusco ${ }^{13,14}$, F. Gargano ${ }^{14}$, N. Gehrels ${ }^{19}$, N. Giglietto ${ }^{13,14}$, P. Giommi ${ }^{31}$, F. Giordano ${ }^{13,14}$, M. Giroletti ${ }^{32}$, T. Glanzman ${ }^{3}$, G. Godfrey ${ }^{3}$, I. A. Grenier ${ }^{5}$, M.-H. Grondin $^{29}$, S. Guiriec ${ }^{33}$, D. Hadasch ${ }^{17}$, Y. Hanabata ${ }^{34}$, A. K. Harding ${ }^{19}$, E. Hays ${ }^{19}$, G. L. Israel ${ }^{35}$, G. Jóhannesson ${ }^{36}$,

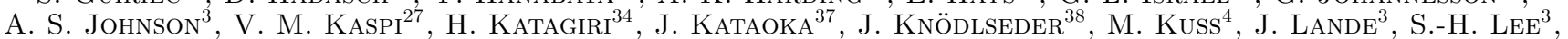

M. Lemoine-Goumard ${ }^{29}$, F. Longo ${ }^{6,7}$, F. Loparco ${ }^{13,14}$, M. N. Lovellette ${ }^{1}$, P. Lubrano ${ }^{11,12}$, A. Makeev ${ }^{1,22}$, M. Marelli ${ }^{18}$, M. N. Mazziotta ${ }^{14}$, J. E. McEnery ${ }^{19,39}$, J. Mehault ${ }^{40}$, P. F. Michelson ${ }^{3}$, T. Mizuno ${ }^{34}$, A. A. Moiseev ${ }^{20,39}$, C. Monte $^{13,14}$, M. E. Monzani ${ }^{3}$, A. Morsellit $^{41}$, I. V. Moskalenko ${ }^{3}$, S. Murgia ${ }^{3}$, M. Naumann-Godo ${ }^{5}$, P. L. Nolan ${ }^{3}$, E. $\mathrm{Nuss}^{40}$, T. Ohsugi ${ }^{42}$ A. OKumura ${ }^{43}$, N. Omodei ${ }^{3}$, E. Orlando $^{44}$, J. F. Ormes ${ }^{45}$, M. Ozaki ${ }^{43}$, D. Paneque ${ }^{3}$, D. Parent ${ }^{1,22}$, M. Pepe ${ }^{11,12}$, M. Pesce-Rollins ${ }^{4}$, F. Piron $^{40}$, T. A. Porter $^{3}$, S. Rainò ${ }^{13,14}$, R. Rando ${ }^{9,10}$, M. Razzano ${ }^{4}$, N. Rea ${ }^{17}$, A. Reimer ${ }^{46,3}$, O. Reimer ${ }^{46,3}$, T. RePoseur ${ }^{29}$, S. Ritz $^{28}$, H. F.-W. Sadrozinski ${ }^{28}$, P. M. Saz Parkinson ${ }^{28}$, C. Sgrò ${ }^{4}$, E. J. Siskind ${ }^{47}$, D. A. Smith ${ }^{29}$, G. Spandre $^{4}$, P. Spinelli ${ }^{13,14}$, M. S. Strickman ${ }^{1}$, H. TAKahashi ${ }^{42}$, T. TAnaka ${ }^{3}$, J. B. Thayer ${ }^{3}$, D. J. ThOmpson ${ }^{19}$, L. Tibaldo ${ }^{9,10,5,48}$, D. F. Torres ${ }^{17,49}$, G. Tosti ${ }^{11,12}$, A. Tramacere ${ }^{3,50,51}$, E. Troja ${ }^{19,52}$, Y. UChiYama ${ }^{3}$, T. L. Usher ${ }^{3}$, J. Vandenbroucke ${ }^{3}$, V. Vasileiou ${ }^{20,21}$, G. Vianello ${ }^{3,50}$, V. Vitale ${ }^{41,53}$, A. P. Waite ${ }^{3}$, B. L. Winer ${ }^{54}$, K. S. Wood ${ }^{1}$, Z. YANG ${ }^{23,24}$, M. ZIEGLER ${ }^{28}$

Draft version October 30, 2018

\section{ABSTRACT}

We report on the search for $0.1-10 \mathrm{GeV}$ emission from magnetars in 17 months of Fermi Large Area Telescope (LAT) observations. No significant evidence for gamma-ray emission from any of the currently-known magnetars is found. The most stringent upper limits to date on their persistent emission in the Fermi energy range are estimated between $\sim 10^{-12}-10^{-10} \mathrm{erg} \mathrm{s}^{-1} \mathrm{~cm}^{-2}$, depending on the source. We also searched for gamma-ray pulsations and possible outbursts, also with no significant detection. The upper limits derived support the presence of a cut-off at an energy below a few $\mathrm{MeV}$ in the persistent emission of magnetars. They also show the likely need for a revision of current models of outer gap emission from strongly magnetized pulsars, which, in some realizations, predict detectable $\mathrm{GeV}$ emission from magnetars at flux levels exceeding the upper limits identified here using the Fermi-LAT observations.

Subject headings: stars: magnetars - X-rays: stars

\footnotetext{
${ }^{1}$ Space Science Division, Naval Research Laboratory, Washington, DC 20375, USA

${ }^{2}$ National Research Council Research Associate, National Academy of Sciences, Washington, DC 20001, USA

${ }^{3}$ W. W. Hansen Experimental Physics Laboratory, Kavli Institute for Particle Astrophysics and Cosmology, Department of Physics and SLAC National Accelerator Laboratory, Stanford University, Stanford, CA 94305, USA

${ }^{4}$ Istituto Nazionale di Fisica Nucleare, Sezione di Pisa, I-56127 Pisa, Italy

${ }^{5}$ Laboratoire AIM, CEA-IRFU/CNRS/Université Paris Diderot, Service d'Astrophysique, CEA Saclay, 91191 Gif sur Yvette, France

${ }^{6}$ Istituto Nazionale di Fisica Nucleare, Sezione di Trieste, I-34127 Trieste, Italy

${ }^{7}$ Dipartimento di Fisica, Università di Trieste, I-34127 Trieste, Italy

8 Rice University, Department of Physics and Astronomy, MS-108, P. O. Box 1892, Houston, TX 77251, USA

${ }^{9}$ Istituto Nazionale di Fisica Nucleare, Sezione di Padova, I-35131 Padova, Italy

${ }^{10}$ Dipartimento di Fisica "G. Galilei", Università di Padova, I-35131 Padova, Italy

${ }^{11}$ Istituto Nazionale di Fisica Nucleare, Sezione di Perugia, I-06123 Perugia, Italy

12 Dipartimento di Fisica, Università degli Studi di Perugia, I-06123 Perugia, Italy
}

${ }^{13}$ Dipartimento di Fisica "M. Merlin" dell'Università e del Politecnico di Bari, I-70126 Bari, Italy

${ }^{14}$ Istituto Nazionale di Fisica Nucleare, Sezione di Bari, 70126 Bari, Italy

15 Laboratoire Leprince-Ringuet, École polytechnique, CNRS/IN2P3, Palaiseau, France

${ }^{16}$ Department of Physics, University of Washington, Seattle, WA 98195-1560, USA

17 Institut de Ciencies de l'Espai (IEEC-CSIC), Campus UAB, 08193 Barcelona, Spain

18 INAF-Istituto di Astrofisica Spaziale e Fisica Cosmica, I-20133 Milano, Italy

19 NASA Goddard Space Flight Center, Greenbelt, MD 20771, USA

${ }^{20}$ Center for Research and Exploration in Space Science and Technology (CRESST) and NASA Goddard Space Flight Center, Greenbelt, MD 20771, USA

${ }^{21}$ Department of Physics and Center for Space Sciences and Technology, University of Maryland Baltimore County, Baltimore, MD 21250, USA

22 George Mason University, Fairfax, VA 22030, USA

${ }^{23}$ Department of Physics, Stockholm University, AlbaNova, SE-106 91 Stockholm, Sweden

24 The Oskar Klein Centre for Cosmoparticle Physics, AlbaNova, SE-106 91 Stockholm, Sweden

${ }^{25}$ Royal Swedish Academy of Sciences Research Fellow, funded by a grant from the K. A. Wallenberg Foundation 


\section{INTRODUCTION}

Magnetars are isolated neutron stars whose emission is thought to be powered by their magnetic energy. They are discovered either through their bursting activity (and in this case named Soft Gamma Repeaters; SGRs; Kouveliotou et al. 1998) or by their strong persistent soft X-ray emission (then named Anomalous X-ray Pulsars; AXPs; Mereghetti \& Stella 1995). In recent years SGRs and AXPs have been recognized as part of the magnetar class, with the discovery of many AXPs and SGRs showing common characteristics and properties (e.g. Kaspi et al. 2003; Rea et al. 2009; Mereghetti et al. 2009; Israel et al. 2010).

${ }^{26}$ Dipartimento di Fisica, Università di Udine and Istituto Nazionale di Fisica Nucleare, Sezione di Trieste, Gruppo Collegato di Udine, I-33100 Udine, Italy

27 Department of Physics, McGill University, Montreal, PQ, Canada H3A 2 T8

28 Santa Cruz Institute for Particle Physics, Department of Physics and Department of Astronomy and Astrophysics, University of California at Santa Cruz, Santa Cruz, CA 95064, USA

29 Université Bordeaux 1, CNRS/IN2p3, Centre d'Études Nucléaires de Bordeaux Gradignan, 33175 Gradignan, France

30 Osservatorio Astronomico di Trieste, Istituto Nazionale di Astrofisica, I-34143 Trieste, Italy

31 Agenzia Spaziale Italiana (ASI) Science Data Center, I-00044 Frascati (Roma), Italy

32 INAF Istituto di Radioastronomia, 40129 Bologna, Italy

33 Center for Space Plasma and Aeronomic Research (CSPAR), University of Alabama in Huntsville, Huntsville, AL 35899, USA

34 Department of Physical Sciences, Hiroshima University, Higashi-Hiroshima, Hiroshima 739-8526, Japan

35 Osservatorio Astronomico di Roma, I-00040 Monte Porzio Catone (Roma), Italy

36 Science Institute, University of Iceland, IS-107 Reykjavik, Iceland

37 Research Institute for Science and Engineering, Waseda University, 3-4-1, Okubo, Shinjuku, Tokyo, 169-8555 Japan

38 Centre d'Étude Spatiale des Rayonnements, CNRS/UPS, BP 44346, F-30128 Toulouse Cedex 4, France

39 Department of Physics and Department of Astronomy, University of Maryland, College Park, MD 20742, USA

${ }^{40}$ Laboratoire de Physique Théorique et Astroparticules, Université Montpellier 2, CNRS/IN2P3, Montpellier, France

${ }^{41}$ Istituto Nazionale di Fisica Nucleare, Sezione di Roma "Tor Vergata", I-00133 Roma, Italy

${ }^{42}$ Hiroshima Astrophysical Science Center, Hiroshima University, Higashi-Hiroshima, Hiroshima 739-8526, Japan

43 Institute of Space and Astronautical Science, JAXA, 3-1-1 Yoshinodai, Sagamihara, Kanagawa 229-8510, Japan

${ }^{44}$ Max-Planck Institut für extraterrestrische Physik, 85748 Garching, Germany

45 Department of Physics and Astronomy, University of Denver, Denver, CO 80208, USA

46 Institut für Astro- und Teilchenphysik and Institut für Theoretische Physik, Leopold-Franzens-Universität Innsbruck, A-6020 Innsbruck, Austria

47 NYCB Real-Time Computing Inc., Lattingtown, NY 115601025, USA

48 Partially supported by the International Doctorate on Astroparticle Physics (IDAPP) program

49 Institució Catalana de Recerca i Estudis Avançats (ICREA), Barcelona, Spain

50 Consorzio Interuniversitario per la Fisica Spaziale (CIFS), I-10133 Torino, Italy

${ }^{51}$ INTEGRAL Science Data Centre, CH-1290 Versoix, Switzerland

52 NASA Postdoctoral Program Fellow, USA

53 Dipartimento di Fisica, Università di Roma "Tor Vergata", I-00133 Roma, Italy

54 Department of Physics, Center for Cosmology and AstroParticle Physics, The Ohio State University, Columbus, OH 43210, USA
Their X-ray luminosities are typically $10^{33}-$ $10^{36} \mathrm{erg} \mathrm{s}^{-1}$. They have spin periods between $2-12 \mathrm{~s}$ and period derivatives in the $10^{-13}-10^{-11}$ Brange. In most of the cases, the magnetic fields derived from their spin periods and period derivatives, assuming only magnetic dipolar losses as is usually done for normal pulsars, are inferred to be $\sim 10^{14}-10^{15}$ Gauss. These high B fields, in particular their toroidal components, impose tremendous stresses on neutron star crusts, and thereby are believed to be the ultimate energy source of magnetar emission (Duncan \& Thompson 1992; Thompson \& Duncan 1993). The strong soft X-ray emission can be empirically modeled with a black body $(k T \sim 0.3-0.7 \mathrm{keV})$, plus a power law ( $\Gamma \sim 1.5-4$; see e.g. Mereghetti 2008 for a review), although recently more physically based models have been developed to account for their X-ray spectra (e.g. Lyutikov \& Gavrill 2006; Fernandez \& Thompson 2007; Rea et al. 2008; Zane et al. 2009). In the past years, magnetars have been discovered as persistent hard non-thermal X-ray sources, emitting up to $\sim 250 \mathrm{keV}$ (e.g. Kuiper et al. 2004, 2006; Götz et al. 2006; den Hartog et al. 2008; Rea et al. 2009; Enoto et al. 2010).

Our current knowledge of their spectra at much higher energies $(>0.5 \mathrm{MeV})$ is very limited. Archival studies of COMPTEL observations were used to place upper limits on the emission of a few magnetars in the $0.75-30 \mathrm{MeV}$ range, of $\sim 10^{-10} \mathrm{erg} \mathrm{s}^{-1} \mathrm{~cm}^{-2}$ at a $2 \sigma$ level (Kuiper et al. 2006). Very poor so far is the knowledge concerning their behavior at energies $>30 \mathrm{MeV}$ (Heyl \& Hernquist 2005), a band of interest given model predictions of measurable synchrotron/curvature emission (Chang \& Zheng 2001; Zhang \& Cheng 2002).

The Large Area Telescope (LAT), the main instrument on board the Fermi Gamma-ray Space Telescope, launched on 2008 June 11th, is the most sensitive telescope to date in the $\mathrm{GeV}$ energy range. We present in this Letter results of a search for emission in the $\mathrm{GeV}$ domain from the first 17 months of Fermi-LAT observations of magnetars ${ }^{1}$.

\section{OBSERVATION AND DATA REDUCTION}

The data analyzed here were taken in survey mode with the Fermi Large Area Telescope, from 4 August 2008 until 1 January 2010. The Fermi-LAT telescope is sensitive to photons with energies from about $20 \mathrm{MeV}$ to more than $300 \mathrm{GeV}$ and uses the pair conversion technique. The direction of an incident photon is derived by tracking the electron-positron pair in a high-resolution converter tracker, and the energy of the pair is measured with a $\mathrm{CsI}(\mathrm{Tl})$ crystal calorimeter. The Fermi-LAT has an on-axis effective area of $8000 \mathrm{~cm}^{2}$, a $2.4 \mathrm{sr}$ field of view, and an angular resolution of $\sim 0.6^{\circ}$ at $1 \mathrm{GeV}$ (for events converting in the front section of the tracker). Furthermore, an anti-coincidence detector identifies the background of charged particles (Atwood et al. 2009).

We analyzed the data using the Fermi Science Tools v9r15 package. Events from the "Pass 6 Diffuse" event class are selected, i.e. the event class with the greatest

${ }^{1}$ We note that during the submission phase of this work another paper has been published reporting upper limits on one of the sources reported in this paper (Sasmaz Mus \& Gogus 2010). 
TABLE 1

Fermi-LAT UPPER LIMITS ON MAGNETARS OBTAINED FROM LIKELIHOOD ANALYSIS.

\begin{tabular}{lccccccccc}
\hline \hline \multicolumn{1}{c}{ Source } & $\begin{array}{c}\mathrm{d}^{*} \\
\mathrm{kpc}\end{array}$ & $\begin{array}{c}\log (\mathrm{B}) \\
\mathrm{Gauss}\end{array}$ & $\begin{array}{c}\log \left(\mathrm{L}_{\mathrm{X}}\right)^{*} \\
\mathrm{erg} \mathrm{s}^{-1}\end{array}$ & $\begin{array}{c}\log \left(\mathrm{L}_{\mathrm{rot}}\right) \\
\mathrm{erg} \mathrm{s}^{-1}\end{array}$ & $\mathrm{TS}$ & $\begin{array}{c}0.1-10 \mathrm{GeV} \\
(\Gamma=2.5)\end{array}$ & $\begin{array}{c}0.1-1 \mathrm{GeV} \\
(\Gamma=1.5)\end{array}$ & $\begin{array}{c}\text { 1-10 GeV } \\
(\Gamma=3.5)\end{array}$ & $\begin{array}{c}\text { 1FGL srcs } \\
\text { within } 3^{\circ}\end{array}$ \\
\hline 1E 1048.1-5937 & 3.0 & 14.78 & 34.00 & 33.90 & 0.0 & $<5.3(12.0)$ & $<3.9(7.7)$ & $<1.7(0.7)$ & 7 \\
SGR 1900+14 & 15 & 14.81 & 35.44 & 34.34 & 0.0 & $<0.4(0.9)$ & $<0.8(2.0)$ & $<0.6(0.2)$ & 5 \\
SGR 0418+5729 & 2.0 & $<12.70$ & 31.77 & $<29.47$ & 2.3 & $<0.4(0.9)$ & $<0.2(0.4)$ & $<0.1(0.04)$ & 2 \\
SGR 1806-20 & 8.7 & 15.15 & 35.21 & 34.40 & 2.8 & $<0.6(1.4)$ & $<0.5(0.9)$ & $<0.12(0.05)$ & 1 \\
4U 0142+614 & 5.0 & 14.11 & 35.32 & 32.10 & 3.6 & $<0.9(2.0)$ & $<0.5(0.9)$ & $<0.3(0.11)$ & 1 \\
1E 1841-045 & 8.5 & 14.85 & 35.34 & 32.99 & 7.5 & $<3.0(6.0)$ & $<6.3(13.0)$ & $<2.4(0.92)$ & 8 \\
XTE J1810-197 & 4.0 & 14.46 & 33.58 & 33.60 & 13.1 & $<5.0(10.0)$ & $<12.0(23.0)$ & $<2.0(0.7)$ & 7 \\
1E 2259+586 & 3.0 & 13.76 & 34.43 & 31.70 & 15.6 & $<1.7(3.9)$ & $<0.6(1.0)$ & $<0.63(0.24)$ & 2 \\
SGR 0501+4516 & 5.0 & 14.23 & 34.77 & 33.49 & 16.3 & $<1.9(4.3)$ & $<0.6(1.0)$ & $<0.5(0.18)$ & 1 \\
1RXS J1708-4009 & 8.0 & 14.67 & 35.27 & 32.75 & 32.1 & $<10.0(20.0)$ & $<5.0(9.0)$ & $<9.0(4.0)$ & 8 \\
CXOU J1647-4552 & 5.0 & 14.20 & 34.41 & 31.89 & 33.7 & $<10.0(20.0)$ & $<10.0(20.0)$ & $<19.0(7.2)$ & 7 \\
SGR 1627-41 & 11 & 14.34 & 33.39 & 34.63 & 36.0 & $<20.0(50.0)$ & $<20.0(30.0)$ & $<5.0(2.0)$ & 8 \\
1E 1547-5408 & 9.0 & 14.32 & 34.16 & 35.00 & 36.2 & $<10.0(20.0)$ & $<7.9(16.0)$ & $<2.1(0.8)$ & 6 \\
& & & & & & & & & 6
\end{tabular}

Note. - Properties of the magnetars studied in this work ordered by the measured TS values derived from the binned analysis (for further info on the first 4 columns see Mereghetti (2008) and reference therein; Rea et al. (2009, 2010) for the newly discovered SGR 0501+4516 and SGR 0418+5729, respectively). The GeV upper limits are reported at $95 \%$ confidence level (see Sect. 5 for details). Fluxes are in units of $10^{-11} \mathrm{erg} \mathrm{s}^{-1} \mathrm{~cm}^{-2}$ (or $10^{-8}$ photons $\mathrm{cm}^{-2} \mathrm{~s}^{-1}$ for numbers in brackets). The last 4 sources and 1E 1841-045 are discussed in detail in the text. ${ }^{*}$ Note that most of the sources have very variable X-ray luminosities, and very uncertain distances, hence those values should be taken as indicative.

purity of gamma rays, having the most stringent background rejection (Atwood et al. 2009). The "Pass 6 v3 Diffuse" instrument response functions (IRFs) are applied in the analysis. For each analyzed source we select events with energy E> $100 \mathrm{MeV}$ in a circular region of interest $(\mathrm{ROI})$ of $10^{\circ}$ radius. The good time intervals are defined such that the ROI does not go below the gammaray-bright Earth limb (defined at $105^{\circ}$ from the Zenith angle), and that the source is always inside the LAT field of view, namely in a cone angle of $66^{\circ}$.

\section{LIKELIHOOD ANALYSIS AND RESULTS}

Gamma-ray emission was analyzed at the positions of all the magnetars known to date, excluding yet unconfirmed candidates. Extragalactic magnetars located in the Large and Small Magellanic Clouds are also excluded due to their large distances and the difficulty of resolving them from their host galaxies (see Abdo et al. 2010, 2010a). See Table 1 for the 13 selected magnetars.

The Test Statistic was employed to evaluate the significance of the gamma-ray fluxes coming from the magnetars. The TS value is used to assess the goodness of a fit, and it is defined as twice the difference between the loglikelihood function maximized by adjusting all parameters of the model, with and without the source, and under the assumption of a precise knowledge of the Galactic and extragalactic diffuse emission. A TS $=25$ roughly corresponds to a $4.6 \sigma$ detection significance (Abdo et al. 2010b).

Binned and unbinned likelihood analysis are applied on the data, using the official tool (gtlike) released by the Fermi-LAT collaboration. The binned likelihood uses events selected in a square inscribed inside the circular ROI (see $\S 2$ ), aligned with celestial coordinates.

For each magnetar, a spectral-spatial model containing diffuse and point-like sources is created, and the parameters are obtained from a maximum likelihood fit to the data. For the Galactic diffuse emission we use the spectral-spatial model "gll_iem_v02.fit", used by the Fermi collaboration to build the First Fermi
Source Catalog (Abdo et al. 2010b; 1FGL hereafter). The extragalactic diffuse emission was modeled as an isotropic emission using the spectrum described in the "isotropic_iem_v02.txt" file ${ }^{2}$. This spectrum also takes into account the residual background of charged particles in the LAT.

In the spectral-spatial model of each magnetar we fixed its position at the localization determined by $\mathrm{X}$ ray observations (in all cases with uncertainties $<2^{\prime \prime}$; see Mereghetti 2008 and the McGill catalog ${ }^{3}$ ), and also included all the point-like sources from the 1FGL list closer than $15^{\circ}$. Each of those point sources was modeled with a simple power-law, with the exceptions of the pulsars closer than $3^{\circ}$ from the magnetars, for which a powerlaw with an exponential cut-off was used. The spectral parameters of those sources were fixed at the 1FGL values or those from the Fermi-LAT First Pulsar Catalog (Abdo et al. 2010c), while the flux parameters of all the point-like sources closer than $3^{\circ}$ to the magnetar were left free in the likelihood fit (see also Table 1, last column).

We modeled the magnetar emission using power-law spectral distributions with two free parameters: the flux and spectral index. The likelihood ratio test indicated values of TS less than 25 for most of the analyzed magnetars (see Table 1). For 1RXS J1708-4009, CXOU J1647-4552, and 1E 1547-5408 the calculated TS values were in the range $25-50$, while SGR 1627-41 and 1E 1841-045 had TS>70. The latter cases are addressed in Sect. 4 .

For those magnetars for which X-ray outbursts were detected during the Fermi-LAT observing period (namely SGR 0501+4516, SGR 0418+5729 and 1E 1547-5408; e.g. Rea et al. 2009; Esposito et al. 2010; Israel et al. 2010), we re-ran the analysis considering subsets of data taken one day, one week or two weeks around the peaks of their X-ray outbursts. All TS values during those outbursts were $<25$.

2 All the data, software, and diffuse models used for this analysis are available from the Fermi Science Support Center. http://fermi.gsfc.nasa.gov/ssc/

${ }^{3}$ www.physics.mcgill.ca/ pulsar/magnetar/main.html 

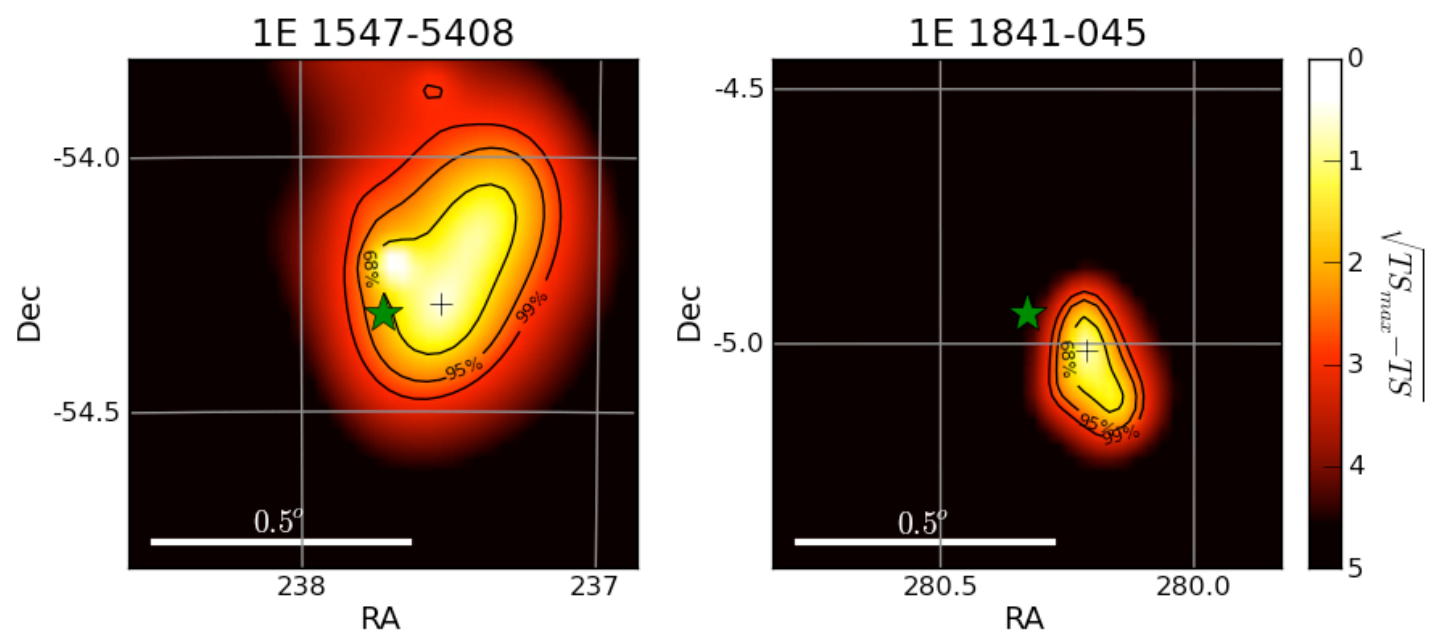

FIG. 1.- Test Statistic maps of the Fermi-LAT fields of 1E 1547-5408 and 1E 1841-045 (RA and Dec are referenced at J2000). The green stars represent the X-ray position of each magnetar. TS max is the maximum TS value inferred around the two magnetars, measured in the position labelled by the crosses. Solid lines are the positional confidence levels around the maximum TS value in each field of view. See text for details.

\section{SOURCES WITH HIGH TS VALUES}

Given the relatively high TS values found in five cases by the likelihood analysis, we checked whether the Xray positions of these magnetars are compatible with the most probable origin of the gamma-ray excesses. For this purpose, we performed a localization process similar to the one used for the 1FGL catalog, using the pointlike tool, which returns the TS map around each source, where the TS is calculated at any putative source position. (see Fig. 1 for two examples of these maps, around 1E 1547-5408 and 1E 1841-045). This tool is applied leaving as free the spectral parameters of the modeled sources within $1^{\circ}$ of each magnetar. The results for the magnetar positions with TS $>25$ are summarized below. We remember here that all these high-TS sources are in the inner Galaxy close to the Galactic plane, where the diffuse emission is strong and highly structured, and this could affect our results.

\section{1. $1 E 1841-045$}

The gtlike analysis of 1E 1841-045 resulted in a high TS value $(>70)$. In this case the pointlike TS map showed a new source very close to the magnetar at an angular distance of $0.11^{\circ}\left(\mathrm{RA}=280.23^{\circ}\right.$, Dec $=-4.99^{\circ}$. See Fig. 1 right panel). This source is not present in the 1FGL catalog, probably due to the longer time interval analyzed here (17 months vs. 11 months for $1 F G L)$. The TS value of $1 \mathrm{E} 1841-045$ falls below 25 when the new source is added to the spectral-spatial model used for the likelihood analysis, and thus we find no evidence to claim the magnetar as a gamma-ray emitter.

\subsection{SGR 1627-41}

The pointlike analysis for SGR 1627-41 indicated that the position of the magnetar was not a maximum of TS when the coordinates of the modeled source were optimized in the fit. In particular, we found that the high TS derived by the gtlike analysis could have been caused by the presence of the rather strong unidentified source (1FGL J1636.4-47371), which lies as close as $0.12^{\circ}$ from the magnetar (although positionally incompatible with it). If the spectral parameters of the modeled 1FGL sources are held fixed at their values in the 1FGL catalog, SGR 1627-41 ends up having a TS 36 . This is what is reported in Table 1. While this is still greater than 25, the flatness of the TS map around this source suggests that in this region the diffuse Galactic emission could be underestimated by the model adopted in the likelihood analysis.

\subsection{RXS J1708-4009 and CXOUJ1647-4552}

The gtlike analyses of these two magnetars resulted in TS values of $\sim 30$ for both sources. For each source we performed a pointlike analysis which in both cases indicated that the position of the two magnetars were not a maximum of TS when the coordinates of the modeled source were optimized in the fit. We cannot exclude that the likelihood excesses of 1RXS J1708-4009 and CXOU J1647-4552 are caused by the uncertainties of the Galactic diffuse model.

\section{4. $1 E 1547-5408$}

$1 \mathrm{E} 1547-5408$ is the only source for which the TS map calculated by pointlike indicated that the position of the magnetar was indeed consistent with a local maximum of TS (see Fig. 1 left panel). In particular, 1E 1547-5408 has a TS 35, and it is observed inside the $95 \%$ positional error contour around the $\mathrm{TS}_{\max }$ of the field. With the current Fermi observations a firm 
association between this excess and 1E 1547-5408 can not be made. Furthermore, we found that the TS of $1 \mathrm{E} 1547-5408$ falls below 20 if the level of the Galactic diffuse emission is increased by only $2 \%$

\section{UPPER LIMITS EVALUATION}

Before starting with the upper limit determination, we note that for all but one magnetar, the local maximum of TS was not coincident with the magnetar position. Furthermore, by increasing the level of the Galactic diffuse emission by $2-4 \%$, all of the TS values determined in $\S 3$ would decrease below 20 . These percentages are well inside the systematics of the assumed Galactic diffuse emission model (see the cases of the supernova remnants W51C and W49, and $\S 4.7$ of the 1FGL catalog; Abdo et al. 2009, 2010d, 2010a).

The discovery of GeV gamma-rays from magnetars would have major implications, hence would require very strong evidence. The evidence so far does not seem to reach more than the circumstantial level, and while the Fermi-LAT exposure continues to accumulate on these sources, we find it appropriate for the time being to report only upper limits.

The upper limits are evaluated by applying the binned likelihood analysis, and using the spectral-spatial models described above. We derived 95\% flux upper limits by fitting a point source at the X-ray magnetar position, for which we increase the flux until the maximum likelihood decreases by $2.71 / 2$ in logarithm.

In the $0.1-10 \mathrm{GeV}$ energy range we fix the photon index value of the magnetars to 2.5 , which is the mean of the photon indexes obtained by the previous likelihood analyses. The other two upper limits are evaluated using spectral index values that mimic a cutoff in the spectrum at $\sim 1 \mathrm{GeV}$, as common in pulsar spectra. Accordingly, in the range $0.1-1 \mathrm{GeV}$ we fix the spectral index to 1.5 , while for $1-10 \mathrm{GeV}$ it is set as 3.5 .

The uncertainties of the Fermi-LAT effective area and of the Galactic diffuse emission are the two main sources of systematics that can affect the evaluation of the upper limits. We estimated the effect of these systematics by repeating the upper limits analysis using modified instrument response functions that bracket the "Pass 6 v3 Diffuse" effective areas, and changing the normalization of the Galactic diffuse model artificially by $\pm 6 \%$. The results of this analysis are reported in Table 1.

\section{TIMING ANALYSIS}

A timing analysis was performed for each of the 13 magnetars studied in this work. With this aim we used the X-ray data available for these objects to build their ephemerides to fold the Fermi-LAT data, or we searched around their X-ray periods when a long-baseline ephemeris could not be derived. In particular, using RXTE and Swift-XRT data, ephemerides ${ }^{55}$ have been derived for $4 \mathrm{U} 0142+614$, 1E 2259+586, 1E 1048.1-5937, 1RXS J1708 -4009, 1E 1841-045, 1E 1547-5408, and SGR 0501+4516 (Dib et al. in prep; Israel et al. 2010; Bernardini et al. in prep; Rea et al. 2009; Rea et al. in prep.). For each of the other magnetars, an ephemeris

\footnotetext{
55 Only in a few cases a single ephemeris could be derived over the entire time-baseline, while in other cases 4-5 different ephemerides were needed to cover the whole Fermi-LAT data span.
}

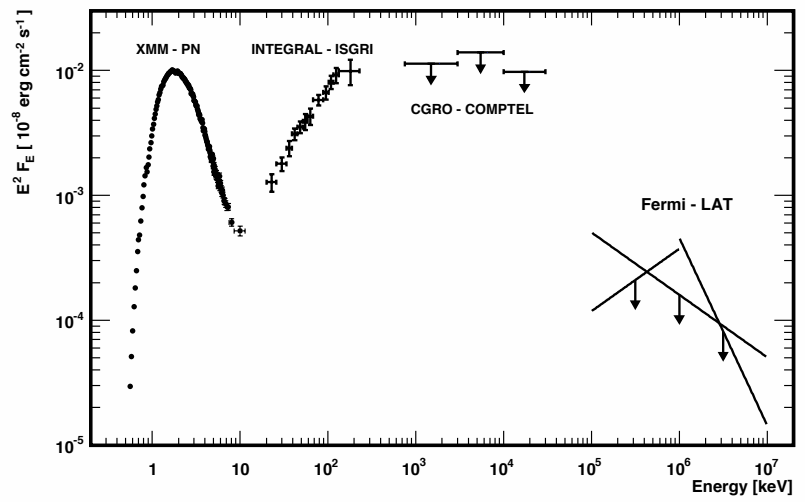

FIG. 2.- Multi-band $\mathrm{E}^{2} \mathrm{~F}_{E}$ spectrum of $4 \mathrm{U} 0142+614$. The $0.1-$ $200 \mathrm{keV}$ data are from $X M M-N$ ewton-PN and INTEGRAL-ISGRI (Rea et al. 2007a; den Hartog et al. 2008; Gonzalez et al. 2010), on which we over-plot the $2 \sigma$ COMPTEL upper limits (den Hartog et al. 2006; Kuiper et al. 2006) and the Fermi-LAT limit (this work) with the assumed power-law spectrum with photon index values of $2.5,1.5$ and 3.5 in the $0.1-10 \mathrm{GeV}, 0.1-1 \mathrm{GeV}$ and $1-10 \mathrm{GeV}$ bands, respectively (see also Table 1 ).

valid throughout the 17 months of Fermi-LAT observations is not derivable either given the paucity of X-ray observations or because the source is too dim to have long-term measurements of its spin period. For these we searched directly in the gamma-ray data, performing a semi-blind search around plausible values of spin period and its derivative (see Mereghetti 2008). With the help of PRESTO software (Ransom 2001), we also tried to improve the signal including trials for the second derivative of the period. No significant signal has been detected either searching in Fermi-LAT data around the X-ray period, or, when possible, folding at the X-ray ephemeris derived from current X-ray monitoring observations.

\section{DISCUSSION}

In this paper we searched for $\mathrm{GeV}$ emission from magnetars using the most sensitive data to date. We did not find evidence beyond reasonable doubt that would allow us to claim the detection of any of these magnetars. In a few cases, putative detections were marked for further studies, but they are not significant enough to claim a new population of gamma-ray emitters.

For all of the studied magnetars we calculated the deepest upper limits derived to date in the $0.1-10 \mathrm{GeV}$ energy range. In Fig. 2 we show the $0.1 \mathrm{keV}-10 \mathrm{GeV}$ multi-band spectrum of $4 \mathrm{U} 0142+614$, the persistent magnetar having the brightest emission and steepest spectral decomposition in the hard X-ray band. Comparing the Fermi-LAT upper limits to the hard X-ray measured fluxes for all the studied magnetars, it is clear that the spectral energy distribution of these objects should necessarily have a cut-off below the $\mathrm{MeV}$ band, as already pointed out for a few sources by COMPTEL observations (den Hartog et al. 2006; Kuiper et al. 2006).

In particular, fitting a log-parabolic functions to the hard X-ray spectrum of $4 \mathrm{U} 0142+614$ (Kuiper et al. 2006; Rea et al. 2007b; den Hartog et al. 2008) as an example resulted in a peak energy of $279_{-41}^{+65} \mathrm{keV}$ (den Hartog et al. 2008). This kind of spectral model has been 
successfully applied to many pulsars such as the Crab or Vela (Kuiper et al. 2001; Massaro et al. 2006a,b; Rea et al. 2007b). Such log-parabolic spectra can be approximately obtained when relativistic electrons are accelerated by some mechanism and competitively cool via synchrotron or by inverse Compton scattering losses. The narrow energy range of each log-parabolic component might then reflect a tight balance between cooling and acceleration in a relatively confined emission locale.

On the other hand, in some cases the hard X-ray tail at $>10 \mathrm{keV}$ can be equally well-modeled with a flat power-law with an exponential cutoff (e.g., den Hartog et al. 2007, 2008) as opposed to a log-parabolic form. One possibility suggested by this is that resonant inverse Compton scattering by a population of highly relativistic electrons energized at altitudes below around ten stellar radii may provide this hard X-ray component of magnetars (see Thompson \& Beloborodov 2005; Baring \& Harding 2007; Nobili, Turolla \& Zane 2008), probably using seed thermal photons emanating from the stellar surface. In this scenario, the Fermi-LAT and COMPTEL spectroscopic constraints, implying a turnover around $200-500 \mathrm{keV}$, profoundly limit a combination of the Lorentz factor of the radiating electrons and the typical viewing angle of the observer (Baring \& Harding 2007). Accordingly, phase-resolved spectroscopy will provide important diagnostics on more refined models of such a scenario (see e.g., den Hartog et al. 2008). Note that Trümper et al. (2010) recently invoked a bulkComptonization, fallback disk model as an alternative, non-magnetar explanation for these tails.

The low Fermi-LAT upper bounds provide interesting constraints on postulated magnetar syn- chrotron/curvature emission from high altitudes. The emerging paradigm for young pulsars that are bright in the $100 \mathrm{MeV}$ - $10 \mathrm{GeV}$ energy range (Abdo et al. $2010 \mathrm{c}$ ) is that they emit due to acceleration in a slotgap or outer-gap potential not far from their light cylinders. Much earlier, Cheng \& Zhang (2001) and Zhang \& Cheng (2002) proposed an outer-gap model for magnetar emission above $30 \mathrm{MeV}$, mediated by pairs created at high altitudes in collisions between X-rays originating on or near the surface, and GeV-band primary photons from electrons accelerated in the gap. Given the nominal Fermi-LAT sensitivity, their model predicted that SGR 1900+14 and five AXPs (see Fig. 5 of Cheng \& Zhang 2001) would have been observable within a year with fluxes of the order of $10^{-7}-10^{-9}$ photons $\mathrm{cm}^{-2} \mathrm{~s}^{-1}$, depending on the assumed parameters. However, FermiLAT does not detect any of these magnetars in 17 months of data. This strong observational diagnostic necessarily forces a revision of the parameter space applicable for the viability of their outer gap model to each magnetar.

The Fermi-LAT Collaboration acknowledges support from a number of agencies and institutes for both development and the operation of the LAT as well as scientific data analysis. These include NASA and DOE in the United States, CEA/Irfu and IN2P3/CNRS in France, ASI and INFN in Italy, MEXT, KEK, and JAXA in Japan, and the K. A. Wallenberg Foundation, the Swedish Research Council and the National Space Board in Sweden. Additional support from INAF in Italy and CNES in France for science analysis during the operations phase is also gratefully acknowledged.

\section{REFERENCES}

Atwood, W. B. et al. 2009, ApJ 697, 1071

Abdo, A. A. et al., 2009, ApJL, 706, 1

Abdo, A. A. et al. 2010, A\&A 512, 7

Abdo, A. A. et al. 2010a, A\&A accepted, arXiv:1008.2127

Abdo, A. A. et al. 2010b, ApJS, 188, 405

Abdo, A. A. et al. 2010c, ApJS, 187, 460

Abdo, A. A. et al., 2010d, ApJ, accepted

Baring, M. G. \& Harding A. K. 2007, Astr. Space Sci, 308, 109

Cheng, K. S. \& Zhang, L. 2001, ApJ 562, 918

Duncan, R. C. \& Thompson C. 1992, ApJ 392, L9

Enoto, T., et al. 2010, ApJ 715, 665

Esposito, P., et al. 2010, MNRAS, 498 in press

Fernandez, R. \& Thompson, C. 2007, ApJ 660, 615

Gonzalez, M. E., et al. 2010, ApJ, 716, 1345

Götz, D., et al. 2006, A\&A, 449, L31

Heyl, J. S., \& Hernquist, L. 2005, MNRAS, 362, 777

den Hartog, P. R. et al 2006, A\&A 451, 587

den Hartog, P. R. et al. 2007, Ap\&SS, 308, 647

den Hartog, P. R. et al. 2008, A\&A 489, 245

Israel, G.L., et al. 2010, MNRAS, in press

Kaspi, V. M., et al. 2003, ApJ 588, L93

Kouveliotou, C. et al. 1998, Nature 393, 235

Kuiper, L. et al. 2001, A\&A, 378, 918

Kuiper, L., Hermsen, W., \& Mendez, M. 2004, ApJ, 613, 1173

Kuiper, L. et al. 2006, ApJ, 645, 556
Lyutikov, M. \& Gavriil, F. P. 2006, MNRAS, 368, 690

Mattox, J. R. et al. 1996, ApJ, 461, 396

Massaro, E. et al. 2006a, A\&A, 459, 859

Massaro, E. et al. 2006b, A\&A, 448, 861

Mereghetti, S. \& Stella, L. 1995, ApJ 442, L17

Mereghetti, S. 2008, A\&AR, 15, 225

Mereghetti, S., et al. 2009, ApJ 696, L74

Nobili, L., Turolla, R. \& Zane, S. 2008, MNRAS, 389, 989

Perna, R., Heyl, J. S., Hernquist, L. E., et al. 2001, ApJ, 557, 18

Ransom, S. 2001, PhD thesis

Rea, N. et al. 2007a, MNRAS 381, 293

Rea, N. et al. 2007b, ApJ 661, L65

Rea, N. et al. 2008, ApJ 686, 1245

Rea, N. et al. 2009, MNRAS 396, 2419

Rea, N. et al. 2010, Science in press (arXiv:1010.2781)

Şaşmaz Muş, S. \& Göğüş, E. 2010, ApJ 723, 100

Thompson, C. \& Duncan, R. C. 1993, ApJ, 408, 194

Thompson, C., Lyutikov, M. \& Kulkarni, S. R. 2002, ApJ, 574, 332

Thompson, C. \& Beloborodov, A. M., 2005, ApJ, 634, 565

Trümper, J. E., Zezas, A., Ertan, Ü. \& Kylafis, N. D. 2010, A\&A, 518,46

Zane, S. et al. 2009, MNRAS, 398, 1403

Zhang, L. \& Cheng, K. S. 2002, ApJ 579, 716 